\title{
Performance of energy expenditure assessment using a chest-worn wireless patch sensor
}

\author{
Nandakumar Selvaraj* and Toai Doan
}

\begin{abstract}
Traditional systems for energy expenditure (EE) assessment are impractical for continuous monitoring in freeliving conditions. The study presents the performance of a chest-worn wireless HealthPatch ${ }^{\circledR}$ sensor for the continuous estimation of EE rate and total energy expenditure (TEE) based on the heart rate and acceleration signals of upper torso. Volunteers $(n=32)$ were attached with patch sensors at three locations on chest, a portable metabolic analyzer, three commercial devices: BodyMediaFIT, Nike+FuelBand and FitBitForce for comparative analysis. Participants carried out a protocol consisted of resting, mild, moderate and intense level of exercises that lasted for $90 \mathrm{~min}$. Analyses of correlation, performance errors and agreement were carried out for the EE rate and TEE values of the patch sensor compared to the metabolic analyzer. The correlation coefficient and mean absolute error of patch sensor's EE rate were $0.94 \pm 0.04$ and $0.67 \pm 0.24(\mathrm{Kcal} / \mathrm{min})$, respectively for the collective three patch locations. The patch sensor offered the most accurate estimates of TEE with least mean absolute percentage error of $<15 \%$, least bias $(0.8 \mathrm{Kcal})$ and narrowest $95 \%$ limits of agreement (-79 $-81 \mathrm{Kcal})$ than the other consumer based wearable sensors.

Index Terms-Energy expenditure, Heart rate, Actigraphy, Wearable sensors, Performance Analysis.
\end{abstract}

\section{INTRODUCTION}

Obesity is a growing health crisis in United States and around the world. Among adults aged 20 years or older in US, more than 1 in 3 are found to be obese, and more than 2 in 3 are overweight and obese combined in 2009-2010 [1]. Obesity/overweight is one of the leading risk factors for major health problems. Poor dietary choices, sedentary life style, and lack of physical activity/exercises primarily disrupt the energy balance, the ratio of energy expended (or burnt) to the energy intake, and cause obesity. Development of tools using wearable sensors to continuously quantify the energy expenditure (EE) rate would allow individuals to accurately track the calories intake and expended.

The energy expenditure is measured in humans using various techniques including whole-body direct calorimetry that quantifies the rate of heat loss in an insulated chamber; non-calorimetry such as doubly labeled water method that estimates the carbon dioxide ( $\mathrm{CO} 2)$ production by measuring the concentration of non-radioactive isotope tracers of oxygen $(\mathrm{O} 2)$ and hydrogen in the body water; and indirect calorimetry that captures the $\mathrm{O} 2$ consumption and exhaled $\mathrm{CO} 2$ production. These commonly used techniques for energy expenditure measurement have their unique challenges and limitations including expensiveness, complex, bulky, uncomfortable and calibration requirements. Most importantly

Authors are with Vital Connect Inc., Campbell, CA 95008, USA, (*corresponding author's e-mail: snandaa@gmail. com) these systems are impractical for continuous monitoring of $\mathrm{EE}$ in free-living conditions.

There are few wearable devices that are shown to provide EE rates with some degree of accuracy during activities based on human movements measured by accelerometer sensors [2]. Smart phone based applications have also emerged to provide EE estimates utilizing the smart phone's accelerometer. But human body acceleration signals obtained from extremities/pockets of clothing could be highly inadequate to track EE estimates particularly during free-living conditions. Further, the acceleration signals alone may not be able to distinguish resting and isometric/static exercises. On the other hand, the combination of changes in heart rate (HR) and body movements has great potential for accurate prediction of $\mathrm{EE}$ rate during static/dynamic exercises and free living conditions [3]. However, monitoring continuous and reliable HR estimates over 24 hours in free-living conditions has been a great challenge.

HealthPatch ${ }^{\circledR}$ sensor is a novel, unobtrusive, wireless patch sensor developed by Vital Connect Inc (VCI) that measures not only the human acceleration signals but also the electrocardiogram, HR and heart rate variability. The patch sensor allows continuous and remote monitoring of $\mathrm{HR}$ and human movements, and provides continuous assessment of EE rate and total daily energy expenditure (TEE). The current study investigates the accuracy of energy expenditure assessment using patch sensors.

\section{MATERIALS AND METHODS}

\section{A. HealthPatch Sensor}

The VCI patch sensor is a disposable adhesive patch sensor worn on the chest that incorporates two surface electrodes with hydrogel on the bottom of the patch, a battery and an electronic module with the embedded processor, tri-axial accelerometer, and Bluetooth Low Energy (BLE) transceiver. The patch sensor facilitates continuous monitoring of single-lead bipolar ECG and human body acceleration signals. The device automatically performs calibration of the triaxial accelerometer to obtain vertical, antero-posterior, and left-right lateral directions during an initial period of standing upright or walking. The firmware algorithms on the electronic module process the raw signals and transmit a stream of physiological measures as encrypted data including heart rate, heart rate variability, respiration rate, skin temperature, posture, step, and fall detection via an encrypted BLE wireless protocol to a relay such as a smartphone, where the live streams of data can be viewed and stored. The physiological monitoring capabilities of patch sensor and its 
clinical validation have been reported elsewhere [4]. This article presents the patch sensor's functionality of providing continuous assessment of EE rate and TEE.

\section{B. Algorithm}

The HR based EE prediction algorithms generally require a calibration procedure that is involved with data collection for sufficient time periods of resting and performing at least moderate level of intense exercises in each individual. The data collected is used to determine an empirical flex point of HR termed as "HRFlex" computed as the average value of the highest HR during rest period and the lowest HR value during exercises. Above the HRflex value, the relationship between HR and EE rate is found to be linear [3].

The major limitations of the widely reported HRflex approach are as follows: The calibration of HRflex requires a test that should be sufficiently long enough to obtain reliable HRflex point in each individual; the reliability of HRflex point using a single calibration test is very limited, since the test needs to be repeated many instances of a day to account for the 24 hour HR variability; the test needs to be performed periodically to take the changes in endurance levels of an individual over time into account; The HRflex is time varying that depends on various factors including, basal heart rate, time of the day, activities involved and demographics; Inaccurate prediction of HRflex point may force to follow the wrong choice of EE prediction models that may lead to significant errors in EE rate; Requirement of lengthy and periodic calibration tests weaken the usability; Resting EE rate estimation as a constant (independent of HR) below HRflex point might also cause significant error in total daily expended energy estimates during the continuous assessment of cumulative EE.

The patch sensor utilizes a machine learning based algorithm that combines the continuous HR and acceleration signals of upper torso for accurate prediction of energy expenditure, does not necessitate any separate calibration procedures, and overcomes the above limitations.

\section{Study Group and Design}

The study recruited 32 volunteers with age of 21-72 years, body mass index (BMI) of 19.6-37.9 and female/male of $17 / 15$. The exclusion criteria included severe skin reaction to adhesives, current pregnancy, uncontrolled hypertension, heart diseases, surgically treated cardiovascular issues, and life threatening arrhythmias. Each participant provided written informed consent and demographic information.

\section{Experimental set-up}

The patch sensors were attached at three recommended locations as shown in Fig. 1 to evaluate the performance of $\mathrm{EE}$ assessment at each location independently. The skin sites on chest were ensured hair free, and prepared with alcohol preparation pads before the sensors attached. The subjects were attached to the Oxycon Mobile (CareFusion, Germany), a wireless portable indirect calorimetry system that measures oxygen uptake $\left(\mathrm{VO}_{2}\right)$ and carbon dioxide production $\left(\mathrm{VCO}_{2}\right)$

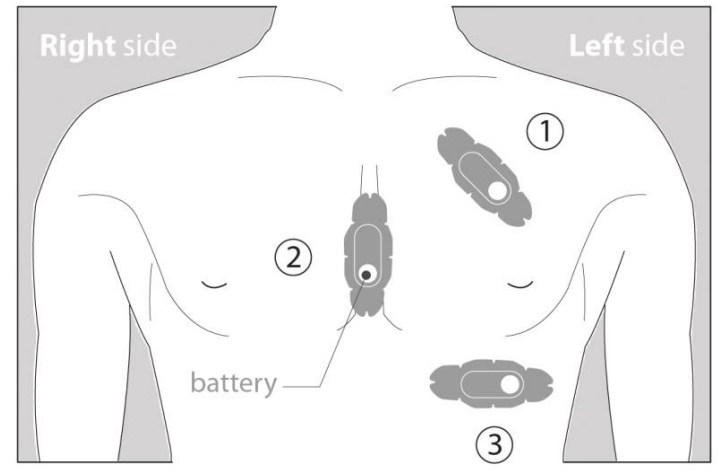

Fig. 1: The disposable HealthPatch ${ }^{\circledR}$ sensor is illustrated with its recommended locations and orientations.

on a breath-to-breath basis, as a reference for performance analysis. Separate calibration procedures were carried out for calibrating the flow-volume control and gas analyzer of the Oxycon according to the manufacturer's guidelines before the test protocol initiated.

Three commercially available consumer based devices: BodyMedia FIT armband (BMF), Nike+Fuelband (NFB), and FitBit Force (FBF) were also attached to the subjects per the manufacturer's recommendations. The BMF armband also known as Sense Wear estimates EE based on the triaxial accelerometer, skin temperature, heat flux, and galvanic skin response of the left upper arm. On the other hand, FBF and NFB devices attached to the wrist estimates EE based on the body movement, incremented steps and distance. The patch sensors and the consumer devices were wirelessly linked to smartphones and the data were collected.

\section{E. Study Design}

The experimental protocol consisted of various tests including resting, activities of daily living, and performing moderate and intense level of exercise that lasted about 90 min collectively. Resting ( $\sim 30 \mathrm{~min})$ included sitting relaxed on a chair, lying down on supine and performing breathing exercises while seated. Simple activities of daily living $(\sim 15$ min) included standing, wiping, sweeping, typewriting and handwriting. Moderate level of exercises ( $\sim 20 \mathrm{~min}$ ) included walking normally on floor, moving and lifting weights, and performing squats. Intense activities $(\sim 20 \mathrm{~min})$ included walking/running on treadmill at speeds varied from $1.7 \mathrm{mph}$ 5 mph with $0 \%-18 \%$ inclinations. Treadmill exercises were terminated for any of these reasons: (i) the subject exceeded $85 \%$ of maximal heart rate, (ii) the subject experienced dizziness, chest pain or injury and (iii) the subject activated emergency stop button of the treadmill. After the treadmill tests, the subject was allowed to recover on a sitting posture ( $\sim 5 \mathrm{~min}$ ). Thus, the protocol was designed to capture a wide range of heart rates, activity and exertion levels to mimic the wide spectrum of energy expenditure rates.

\section{F. Data Analysis}

The EE rate estimates of each patch sensor were compared to that of respective reference EE rates of portable 


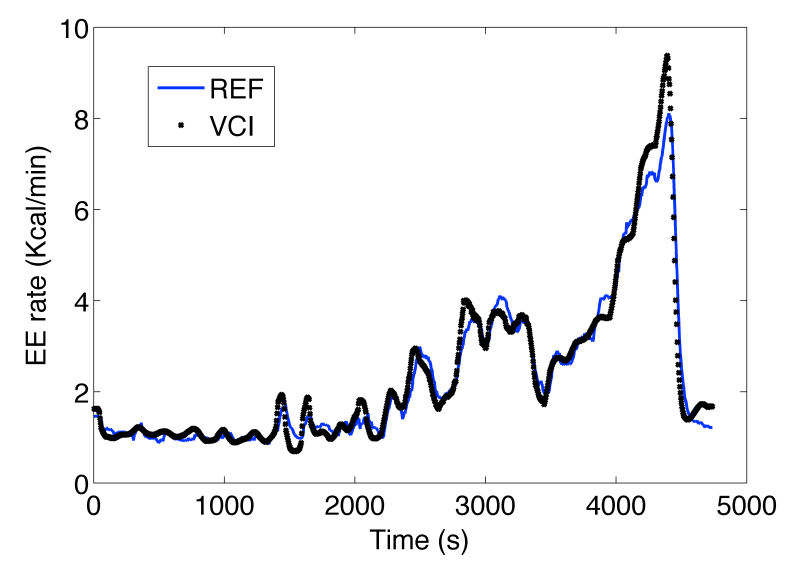

Fig. 2: Comparison of energy expenditure rate estimates of VCI sensor vs the Oxycon, the reference metabolic analyzer, for a sample subject.

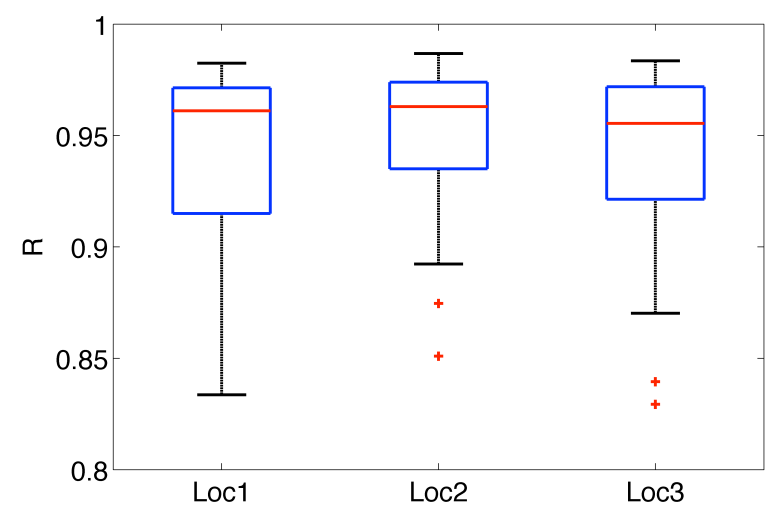

Fig. 3: The box plots of correlation coefficient (R) quantified using the EE rates of reference and VCI sensor in 32 subjects.

metabolic analyzer, and the following performance analyses were carried out for each patch locations. The strength of the linear relationship between predicted EE rates of VCI sensor and true EE rates from Oxycon metabolic analyzer was quantified using the Pearson correlation coefficient (R). Mean absolute error (MAE) and root mean square error (RMSE) were quantified to assess the accuracy of EE rate estimates of the patch sensor. The statistical significance of MAE and RMSE values over the 3 patch locations in 32 subjects was assessed using one-way analysis of variance test. The degree of agreement between the predicted and true EE rates was quantified by the bias, lower and upper limits of agreement with $95 \%$ confidence intervals using BlandAtman plot technique. The above performance measures were also quantified for the collective EE rates from all the 3 patch locations. Further, the accuracies of total expended energy over the 90 min protocol obtained from patch sensors and commercial devices were assessed by calculating the mean absolute percentage error (MAPE), linear correlation and limits of agreement with respect to the TEE values of portable metabolic analyzer. All the sample values are given as mean \pm standard deviation.
TABLE I: Comparison of performance metrics $(n=32)$ for the assessment of energy expenditure rate.

\begin{tabular}{c|c|c|c|c}
\hline \hline Metric & Location & Location & Location & Locations \\
& 1 & 2 & 3 & All \\
\hline \hline MAE & $0.67 \pm 0.24$ & $0.65 \pm 0.18$ & $0.67 \pm 0.17$ & $0.66 \pm 0.20$ \\
RMSE & $0.97 \pm 0.38$ & $0.96 \pm 0.34$ & $0.97 \pm 0.30$ & $0.96 \pm 0.33$ \\
\hline \hline Lower & -1.94 & -2.02 & -1.96 & -1.98 \\
Bias & 0.06 & -0.05 & 0.03 & 0.01 \\
Upper & 2.06 & 1.92 & 2.01 & 2.00 \\
\hline \hline
\end{tabular}

MAE, mean absolute error; RMSE, root mean square error; Lower and Upper are the $95 \%$ limits of agreement; The units are in $\mathrm{Kcal} / \mathrm{min}$.

\section{RESULTS}

The predicted energy expenditure rate data of VCI's patch sensor of a sample subject is plotted against the reference EE rate values of Oxycon metabolic analyzer in Fig. 2. The predicted EE rates showed great correspondence to that of reference through distinct phases of protocol including resting, simple, moderate, and intense activities followed by a recover period. Analysis of data in 32 subjects found a strong correlation between the predicted and reference EE rates as $0.94 \pm 0.04,0.95 \pm 0.03,0.94 \pm 0.04$ for the 3 patch locations, respectively (as shown in Fig. 3). The overall correlation was $0.94 \pm 0.04$ for all the patch locations, collectively. Table 1 illustrates the error and agreement analyses of predicted EE rates among each and the collective patch locations. No significant differences $(\mathrm{P}>0.05)$ were noticed in performance measures (R, MAE and RMSE) among the 3 patch locations. The overall MAE and RMSE were $0.66 \pm 0.20 \mathrm{Kcal} / \mathrm{min}$ and $0.96 \pm 0.33 \mathrm{Kcal} / \mathrm{min}$, respectively considering the 3 patch locations collectively. The Bland-Atlman agreement analysis showed a negligible bias of $0.01 \mathrm{Kcal} / \mathrm{min}$ and an excellent $95 \%$ limits of agreement $(-1.98-2.00 \mathrm{Kcal} / \mathrm{min})$ for the prediction of EE rate using the collective locations.

The VCI patch sensor provides the time series of cumulative increase in TEE based on the predicted EE rates. The incremental changes in the predicted TEE followed the reference TEE estimates over time very closely through out the protocol in this subject as shown in Fig. 4. (the same sample data shown in Fig. 2). On the other hand, the NFB and FBF devices provide under estimation of TEE during resting/no activities and overestimation during moderateto-intense activities (Fig. 4). The incremental changes in TEE showed a correlation of $0.998 \pm 0.002,0.991 \pm 0.006$, $0.970 \pm 0.018$, and $0.986 \pm 0.019$ for VCI, BMF, NFB, FBF sensors, respectively. The performance metrics of correlation, MAPE (Fig. 5) and agreement for the final estimates of TEE after performing the protocol are given in Table 2 for the VCI and consumer devices. The results showed that the VCI sensor offered the least measurement error of $<15 \%$, while the Nike+Fuelband sensor offered the most MAPE of $\sim 90 \%$. The correlation of the predicted total expended energy was highest in VCI sensor (0.69) and lowest (0.28) in FitBit Force sensor. HealthPatch offered the least bias $(0.8 \mathrm{Kcal})$ and narrowest limits of agreement with $95 \%$ confidence ($79-81 \mathrm{Kcal})$. On the other hand, BodyMediaFit under esti- 




Fig. 4: The comparison of incremental change in TEE over time obtained for the same subject of Fig. 1 using Oxycon (REF), VCI sensor, and three consumer devices.



Fig. 5: The box plots of mean absolute percentage errors (MAPE) obtained from the final TEE estimates of VCI and three consumer devices compared to the reference TEE estimates of Oxycon system in 32 subjects.

mates TEE with bias of $-58 \mathrm{Kcal}$, where as Nike+FuelBand overestimates TEE with a bias of whopping $221 \mathrm{Kcal}$ and FitBit Force sensor offers very broad limits of agreement as shown in Table 2.

\section{Discussion}

The current study investigated the performance of chestworn wireless HealthPatch ${ }^{\circledR}$ sensor for the continuous assessment of EE rate and TEE. The results showed excellent accuracy and agreement for the prediction of EE rates and TEE in a group of 32 individuals that represented balanced gender, and wide range of age and BMI.

A recent study compared the accuracy of few activity based wearable devices for the estimation of EE that ranged from $75 \%$ to $90 \%$ during a 69 min experimental protocol involved with various activities [2]. The EE prediction of [2] and few other previous studies could be too optimistic due to the reasons including: (i) the resting/no activity time period was very low compared to the total time duration of the protocol, (ii) the experimental protocols did not include
TABLE II: Comparison of performance metrics $(n=32)$ for the assessment of final TEE at the end of the protocol.

\begin{tabular}{c|c|c|c|c}
\hline \hline Metric & VCI & BMF & NFB & FBF \\
\hline \hline $\mathrm{R}$ & 0.69 & 0.58 & 0.35 & 0.28 \\
MAPE & $14.5 \pm 10.0$ & $24.7 \pm 11.7$ & $89.7 \pm 53.1$ & $21.3 \pm 12.7$ \\
\hline \hline Lower & -79.2 & -141.5 & -29.3 & -119.5 \\
Bias & 0.8 & -58.4 & 220.9 & 4.2 \\
Upper & 80.8 & 24.7 & 471.1 & 127.9 \\
\hline \hline
\end{tabular}

$\mathrm{R}$, correlation coefficient; MAPE, mean absolute percentage error in \%, Lower and Upper are the $95 \%$ limits of agreement in Kcal.

a recovery phase after performing intense activities, and (iii) demographics of the study group were not diverse in age and BMI from their investigations. Including a recovery phase in the experimental protocol could be very important, since it may represent the time durations with lower/no body movements and higher heart rates that might mimic isometric/static exercises, physical stress or psychological stress. The free-living conditions usually include different EE dynamics and durations of resting, stress, and recovery after activities.

The current study design has included significant time period of resting $(>30 \%)$, a recovery phase after intense activities and a diverse study group. HealthPatch sensor is shown to predict the EE rate very accurately for different dynamics including recovery phase (as shown in Fig. 2) due to the sensor fusion of HR with human movements. On the other hand, the performance of the commercial activity sensors are found to be relatively poor in the current study than reported elsewhere [2]. Thus, activity only based sensors could be inaccurate for isometric exercises and for the prediction of overall total daily energy expenditure. The sports activities such as driving/biking might cause even more significant errors in activity based devices.

In conclusion, the current results show that the VCI Patch sensor provides the most accurate measurement of $\mathrm{EE}$ rate as well as total energy expenditure compared to the other major consumer devices on the market. Such wearable technology might help to provide more awareness about individual's energy restrictions and lead to healthy living.

\section{ACKNOWLEDGMENT}

The author thanks H. Gould for managing various aspects of the field study.

\section{REFERENCES}

[1] K. M. Flegal, M. D. Carroll, B. K. Kit, and C. L. Ogden, "Prevalence of obesity and trends in the distribution of body mass index among us adults, 1999-2010," Jama, vol. 307, no. 5, pp. 491-497, 2012.

[2] J.-M. Lee, Y. Kim, and G. J. Welk, "Validity of consumer-based physical activity monitors." Medicine and science in sports and exercise, 2014.

[3] K. Rennie, T. Rowsell, S. Jebb, D. Holburn, and N. Wareham, "A combined heart rate and movement sensor: proof of concept and preliminary testing study." European journal of clinical nutrition, vol. 54, no. 5, pp. 409-414, 2000.

[4] A. M. Chan, N. Selvaraj, N. Ferdosi, and R. Narasimhan, "Wireless patch sensor for remote monitoring of heart rate, respiration, activity, and falls," in Engineering in Medicine and Biology Society (EMBC), 2013 35th Annual International Conference of the IEEE. IEEE, 2013 , pp. 6115-6118. 